\title{
Even low doses of dexmedetomidine produced obstructive apnea during spinal anesthesia
}

\author{
Chiaki Ono, MD • Michiko Sugita, MD • Yoshihiro Ikuta, MD • \\ Tatsuo Yamamoto, MD
}

Received: 30 May 2017/Revised: 14 August 2017/Accepted: 28 August 2017/Published online: 31 August 2017

(C) Canadian Anesthesiologists' Society 2017

\section{To the Editor,}

Dexmedetomidine (DEX) is a sedative that produces less respiratory depression than midazolam or opioids. ${ }^{1,2}$ We examined whether DEX at $<0.7 \mathrm{ng} \cdot \mathrm{mL}^{-1}$ (an appropriate plasma concentration for sedation in the intensive care unit $)^{3}$ provided a plasma concentration that would sedate surgical patients sufficiently under spinal anesthesia.

The protocol of this study was approved by the Ethics Committee of Kumamoto University Hospital, Kumamoto, Japan and is registered in the UMIN Clinical Trial Registry (UMIN 000013558).

The study included 23 patients who were scheduled for surgery with spinal anesthesia using $0.5 \%$ isobaric bupivacaine (puncture site at L3/4 or L4/5; upper effective level at Th5). In a pilot study, in which DEX was administered at a dose recommended in the package insert (1 $\mu \mathrm{g} \cdot \mathrm{kg}^{-1}$ for ten minutes and then 0.2 $\mu \mathrm{g} \cdot \mathrm{kg}^{-1} \cdot \mathrm{hr}^{-1}$ ), we found that the mean (SD) plasma DEX concentration (DEXC) was $0.78(0.21) \mathrm{ng} \cdot \mathrm{mL}^{-1}$ at the end of surgery. In the present study, DEX was administered intravenously at a dose of $0.5 \mu \mathrm{g} \cdot \mathrm{kg}^{-1}$ for ten minutes and then $0.2 \mu \mathrm{g} \cdot \mathrm{kg}^{-1} \cdot \mathrm{hr}^{-1}$ after sensory blockade was achieved up to the appropriate level. Oxygen at $2 \mathrm{~L} \cdot \mathrm{min}^{-1}$ was administered using a nasal cannula.

Trial registration: www.umin.ac.jp/ctr/. Registered 30 March 2014.

C. Ono, MD · M. Sugita, MD · T. Yamamoto, MD ( $\square)$ Department of Anesthesiology, Kumamoto University Hospital, Kumamoto, Kumamoto, Japan

e-mail: yamyam@kumamoto-u.ac.jp

Y. Ikuta, MD

Surgical Center, Kumamoto University Hospital, Kumamoto, Kumamoto, Japan
The respiratory pattern was evaluated during the entire surgical period by measuring chest and abdominal excursions, airflow at the nose and mouth, snoring sounds at the neck using SAS-3200 (Nihon Kohden, Tokyo, Japan), and measuring the $\mathrm{CO}_{2}$ concentration at the nose and mouth using Capnostream 20 (Nihon Kohden). Apnea was defined as airflow cessation for more than $15 \mathrm{sec}$. Obstructive apnea was defined as airflow cessation while chest and/or abdominal excursions were observed. The sedative effect was estimated using the Observer's Assessment of Alertness/Sedation (OAA/S) score. Patient satisfaction level was evaluated using a five-point scale (5: very satisfied, 1: very unsatisfied) at the time of discharge. Dexmedetomidine concentration and $\mathrm{PaCO}_{2}$ were measured at the end of surgery. Dexmedetomidine concentrations were measured by its manufacturer (Maruishi Pharmaceutical, Osaka, Japan), with the precise measurement method not yet published.

The mean (standard deviation [SD]) patient characteristics for the 21 men and two women were as follows: age 71 (10) yr; height 162 (7) cm; weight 60 (11) $\mathrm{kg}$; body mass index 23.0 (3.6) $\mathrm{kg} \mathrm{m}^{-2}$. The mean (SD) duration of DEX infusions was 49 (31) min. The mean (SD) cumulative dose of DEX was 42.6 (9.5) $\mu \mathrm{g}$, with the DEXC at the end of surgery at $0.47(0.09) \mathrm{ng} \cdot \mathrm{mL}^{-1}$. The minimum OAA/S score was 3.3 (range 1-5). The satisfaction level on the five-point scale was 4.2 (range 2-5). Overall, 17/23 (73.9\%) patients had apnea, with each event being of an obstructive type. At the end of the surgery, the $\mathrm{PaCO}_{2}$ level was 43 (5) $\mathrm{mmHg}$. There was no correlation between the $\mathrm{DEXC}$ and $\mathrm{PaCO}_{2}$ (see Figure, $\mathrm{r}=$ $0.39, P=0.07)$. There was no difference between the DEXC of apnea(+) patients $\left[\left(0.47(0.10) \mathrm{ng} \cdot \mathrm{mL}^{-1}\right]\right.$ and that of apnea $(-)$ patients $\left[0.47(0.09) \mathrm{ng} \cdot \mathrm{mL}^{-1}\right]$ (see Figure, $P=0.9$ by Student's $t$ test). Although the $\mathrm{SpO}_{2}$ 


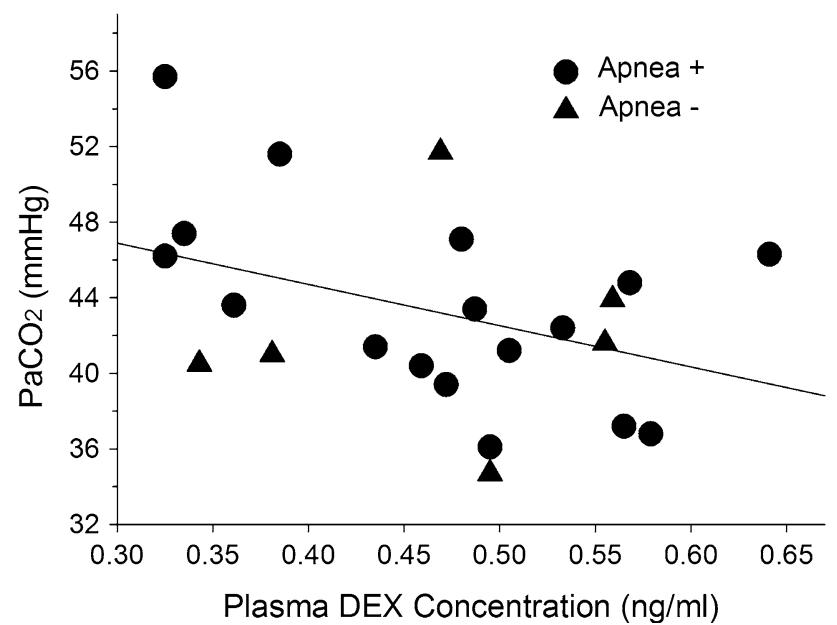

Figure Relation between the plasma dexmedetomidine (DEX) concentration $\left(\mathrm{ng} \cdot \mathrm{mL}^{-1}\right)$ and $\mathrm{PaCO}_{2}(\mathrm{mmHg})$ at the end of the operation. Regression analysis revealed no significant relation between the plasma DEX concentration and $\mathrm{PaCO}_{2}(\mathrm{r}=0.39 ; P=$ 0.07). There was no difference between the plasma DEX concentrations in apnea $(+)$ patients and apnea $(-)$ patients $(P>0.9$, Student's $t$ test $)$. Closed circles $=$ patients with apnea; closed triangles $=$ patients without apnea

decreased to $<90 \%$ in 3 of the 23 patients (13\%) during the operation, every patient restarted breathing spontaneously.

Although the DEXC was relatively low $\left(0.47 \mathrm{ng} \cdot \mathrm{mL}^{-1}\right)$ in this study, the minimum OAA/S score was 3, and the mean (SD) satisfaction level was 4 (1). These data suggest that the DEX regimen used in this study induced an appropriate sedation level for surgical patients under spinal anesthesia. Dexmedetomidine concentration was not directly related to the respiratory effects. Dexmedetomidine produced obstructive apnea in $74 \%$ of patients even at a low DEXC. Although this study lacked a control group, and it is possible that the spinal anesthesia itself was associated with the respiratory changes, ${ }^{4,5}$ we recommend that respiratory pattern monitoring be mandatory in surgical patients under spinal anesthesia during a low-dose DEX infusion.

Conflicts of interest None of the authors had any conflicts of interest.

Editorial responsibility This submission was handled by Dr. Gregory L. Bryson, Deputy Editor-in-Chief, Canadian Journal of Anesthesia.

Funding This study was partly supported by Kumamoto University.

\section{References}

1. Candiotti KA, Bergese SD, Bokesch PM, et al. Monitored anesthesia care with dexmedetomidine: a prospective, randomized, double-blind, multicenter trial. Anesth Analg 2010; 110: 47-56.

2. Hsu YW, Cortinez LI, Robertson KM, et al. Dexmedetomidine pharmacodynamics: part I: crossover comparison of the respiratory effects of dexmedetomidine and remifentanil in healthy volunteers. Anesthesiology 2004; 101: 1066-76.

3. Kamibayashi T, Maze $M$. Clinical uses of $\alpha_{2}$-adrenergic agonists. Anesthesiology 2000; 93: 1345-9.

4. Morley AP, Chung DC, Wong AS, Short TG. The sedative and electroencephalographic effects of regional anaesthesia. Anaesthesia 2000; 55: 864-9.

5. Yamakage $M$, Namiki A, Tsuchida $H$, Iwasaki $H$. Changes in ventilator pattern and arterial oxygen saturation during spinal anaesthesia in man. Acta Anaesthesiol Scand 1992; 36: 569-71. 